\title{
Bioanalysis special focus issue on antibody-drug conjugates
}

\section{"As knowledge emerges from the breadth of antibody-drug conjugates currently in development within the industry, it will help to shape best practices for bioanalysis of antibody-drug conjugates."}

Antibody-drug conjugates (ADCs) contain a cytotoxic drug covalently bound to a monoclonal antibody via a synthetic linker. The concept of an ADC is to deliver the highly potent cytotoxic agent specifically to tumors with minimal impact on normal tissues. This relies on designing the ADC to bind to target antigens that are overexpressed on the surface of the tumor, but minimally expressed on normal tissues. This is followed by internalization, trafficking to the lysosome and cleavage of the linker, resulting in the release of the drug inside the tumor cell. Early ADCs used murine antibodies that were immunogenic, targeted antigens that were not sufficiently selective and carried drugs that lacked potency or used linkers that were too unstable. It has taken time to move forward and has required technological advancements across multiple fields. After more than 25 years we may be on the verge of realizing the promise of ADCs - treating cancer patients without the debilitating side-effects of conventional chemotherapies. There is considerable excitement in the industry with the US FDA approval of Adcetris ${ }^{\circledR}$ (brentuximab vedotin) in 2011 to treat Hodgkin's lymphoma, and the recent approval of Kadcyla $^{\circledR}$ (ado-trastuzumab emtansine) in 2013 to treat women with advanced breast cancers, strongly positive for HER2 [1,101,102]. There is also a robust pipeline of ADCs in preclinical and clinical development [2]. This is therefore an opportune time for Bioanalysis to publish a special issue focusing on ADCs. This issue includes examples of analytical approaches, as well as presenting data from research, nonclinical and clinical phases of ADC development. Examples include MS-based approaches to detect covalent DNA adducts that provide direct evidence for the mechanism of action for a DNA-alkylating ADC [3] and characterization assays to determine drug-to-antibody ratio (DAR) distributions of ADCs in serum/plasma [4]. A nonclinical immunogenicity assessment example includes assays for the detection and epitope characterization of anti-ADC antibodies [5]. Nonclinical and clinical case studies of PK and immunogenicity assays with the recently US FDA-approved ado-trastuzumab emtansine are presented [6,7]. A position paper on ADCs submitted by a working group from the American Association of Pharmaceutical Scientists, including representatives from across the industry and the FDA, discusses factors to be considered for PK and immunogenicity assessment of ADCs and the need to align bioanalytical approaches to facilitate consistent interpretation of data [8].

Bioanalysis of ADCs is challenging compared with small-molecule drugs and protein therapeutics [8,9] because ADCs have complex molecular structures, combining the molecular characteristics of small-molecule drugs with those of protein therapeutics. In addition, ADCs are heterogeneous, because conjugating the drug to the antibody results in a range of DARs with the cytotoxic drug covalently bound at multiple sites in the antibody. The degree of heterogeneity varies with the conjugation chemistry used; conjugation at lysine residues results in somewhat greater heterogeneity than conjugation at reduced interchain cysteine residues. Conjugation at engineered site-specific cysteine residues can be used to produce a homogeneous ADC. However, even for a homogeneous ADC, once in circulation, heterogeneity may arise due to biotransformations [10]. For small-molecule drugs or protein therapeutics, the target species for bioanalysis is relatively straightforward and a single quantitative assay is generally sufficient. In contrast, for ADCs, the situation is more complex, as there are multiple species in circulation that may provide an understanding of clinical safety and efficacy. Therefore, ADC bioanalysis requires strategies to understand the species in circulation and a variety of quantitative assays appropriate for dynamically changing mixtures $[8,9]$.

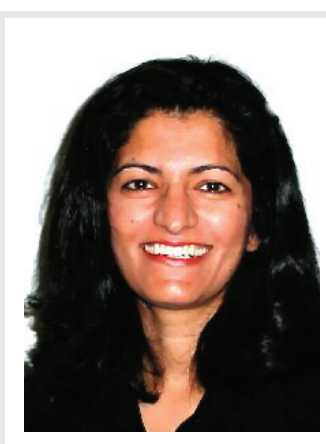

Surinder Kaur

Genentech, I DNA Way, South San Francisco, CA 94080-4990, USA Tel.: + I 6502254776

Fax: +| 6502251998

Email: kaur.surinder@gene.com 
Both ligand-binding methods and LC-MS/MS methods are used to quantify ADCs. There are some limitations to note with either approach. In the case of LC-MS/ MS, an a priori postulated form of the drug released by the ADC in circulation is measured; however, this may not correspond to the major form(s) of the drug released. It is theoretically possible that the released drug contains part of the linker, or that the released drug may bind covalently to plasma peptides or proteins. In other words, the released drug assay may only measure a fraction of the drug that is actually released in circulation. For ligandbinding assays, the calibration standard curve is composed of a reference standard that may not represent the changing DARs in vivo over time. This may be due to biotransformation, differing DAR clearance rates or a combination of both processes. Therefore, the standard curve may not be accurate for the species in vivo at all PK time points. It is critical to assess the accuracy of the assay by testing standards representing the species that are expected to exist or be formed in circulation.

"Moving forward, it will be critical to continue
to explore a diversity of existing and novel
bioanalytical approaches that provide accurate
information, to help answer key questions for
understanding safety and efficacy."

Some methods developed specifically for ADCs combine aspects of ligand binding and MS, such as affinity capture LC-MS, to measure molecular masses of ADCs in vivo [4]. The molecular masses help to determine the DAR distribution and relative abundance of each species. Another hybrid method involves affinity capture and LC-MS/MS to measure antibodyconjugated drug [9]. In this case, the ADC is isolated from serum or plasma by affinity capture and the linker is cleaved to release the drug. Novel methods such as these, designed specifically for use with complex ADCs, can provide additional information that cannot be obtained from conventional approaches alone. Overall, integrated bioanalytical strategies using data from a variety of assay types are used to evaluate the PK of complex ADCs. Each assay provides insights into different aspects of the mixture. Over time, as the relationships between assay data and safety and efficacy are better understood, it may be possible to reduce the number of assays used for PK assessment.

The bioanalysis of ADCs is still new and evolving. Given the limited information that is currently available on ADCs in the clinic, it is not well understood which ADC species correlate best with safety and efficacy. Moving forward, it will be critical to continue to explore a diversity of existing and novel bioanalytical approaches that provide accurate information, to help answer key questions for understanding safety and efficacy. In addition, it will be important for bioanalytical assays to help address questions unique to the development of the ADC platform. For example, the structural stability of different types of linkers in vivo, the stability of functional moieties in the drug or the binding properties of the antibody after conjugation. It may be necessary to develop bioanalytical strategies on a case-bycase basis depending on antibody-linker drug molecular properties. As knowledge emerges from the breadth of ADCs currently in development within the industry, it will help to shape best practices for bioanalysis of ADCs.

\section{Financial \& competing interests disclosure}

The author is employed by Genentech, a member of the Roche group. The author has no other relevant affiliations or financial involvement with any organization or entity with a financial interest in or financial conflict with the subject matter or materials discussed in the manuscript apart from those disclosed.

No writing assistance was utilized in the production of this manuscript.

\section{References}

1 Verma S, Miles D, Gianni L et al. Trastuzumab emtansine for HER2-positive advanced breast cancer. N. Engl. J. Med. 367(19), 1783-1791 (2012).

2 Teicher BA, Chari RV. Antibody conjugate therapeutics: challenges and potential. Clin. Cancer Res. 17(20), 6389-6397 (2011).
3 Thevanayagam L, Bell A, Chakraborty I et al. Novel detection of DNA-alkylated adducts of antibody-drug conjugates with potentially unique preclinical and biomarker applications Bioanalysis 5(9), 1073-1081 (2013).

$4 \mathrm{Xu} \mathrm{K}$, Liu L, Dere R et al. Characterization of the drug-to-antibody ratio distribution for antibody-drug conjugates in plasma/serum Bioanalysis 5(9), 1057-1071 (2013).

5 Hoofring SA, Lopez R, Hock MB et al. Immunogenicity testing strategy and bioanalytical assays for antibody-drug conjugates. Bioanalysis 5(9), 1041-1055 (2013). 
6 Dere RC, Yi J-H, Lei C et al. PK assays for antibody-drug conjugates: case study with ado-trastuzumab emtansine. Bioanalysis 5(9), 1025-1040 (2013).

7 Carrasco-Triguero M, Yi J-H, Dere R et al. Immunogenicity assays for antibody-drug conjugates: case study with ado-trastuzumab emtansine. Bioanalysis 5(9), 1007-1023 (2013).

8 Gorovits B, Alley SC, Bilic S et al. Bioanalysis of antibody-drug conjugates: American Association of Pharmaceutical Scientists
Antibody-Drug Conjugate Working Group position paper. Bioanalysis 5(9), 997-1006 (2013).

9 Kaur S, Xu K, Saad O et al. A perspective on bioanalytical assay strategies for the development of antibody-drug conjugate biotherapeutics. Bioanalysis 5(2), 201-226 (2013).

10 Shen BQ, Xu K, Liu L et al. Conjugation site modulates the in vivo stability and therapeutic activity of antibody conjugates. Nat. Biotechnol. 30(2), 184-189 (2012).

\section{- Websites}

101 US FDA (2011), Adcetris ${ }^{\circledR}$ approval. www.fda.gov/NewsEvents/Newsroom/ PressAnnouncements/ucm268781.htm

102 US FDA (2013), Kadcyla ${ }^{\circledR}$ approval. www.fda.gov/NewsEvents/Newsroom/ PressAnnouncements/ucm340704.htm 